\title{
Existence Results for Nonlinear Boundary Value Problems of Fractional Integrodifferential Equations with Integral Boundary Conditions
}

\author{
Bashir Ahmad ${ }^{1}$ and Juan J. Nieto ${ }^{2}$ \\ ${ }^{1}$ Department of Mathematics, Faculty of Science, King Abdulaziz University, P.O. Box 80203, \\ Jeddah 21589, Saudi Arabia \\ ${ }^{2}$ Departamento de Análisis Matemático, Facultad de Matemáticas, Universidad de Santiago de Compostela, \\ 15782 Santiago de Compostela, Spain
}

Correspondence should be addressed to Bashir Ahmad, bashir_qau@yahoo.com

Received 9 December 2008; Revised 13 January 2009; Accepted 23 January 2009

Recommended by Donal O’Regan

This paper deals with some existence results for a boundary value problem involving a nonlinear integrodifferential equation of fractional order $q \in(1,2]$ with integral boundary conditions. Our results are based on contraction mapping principle and Krasnosel'skiř's fixed point theorem.

Copyright (C) 2009 B. Ahmad and J. J. Nieto. This is an open access article distributed under the Creative Commons Attribution License, which permits unrestricted use, distribution, and reproduction in any medium, provided the original work is properly cited.

\section{Introduction}

In the last few decades, fractional-order models are found to be more adequate than integerorder models for some real world problems. Fractional derivatives provide an excellent tool for the description of memory and hereditary properties of various materials and processes. This is the main advantage of fractional differential equations in comparison with classical integer-order models. Fractional differential equations arise in many engineering and scientific disciplines as the mathematical modelling of systems and processes in the fields of physics, chemistry, aerodynamics, electrodynamics of complex medium, polymer rheology, and so forth, involves derivatives of fractional order. In consequence, the subject of fractional differential equations is gaining much importance and attention. For examples and details, see [1-22] and the references therein. However, the theory of boundary value problems for nonlinear fractional differential equations is still in the initial stages and many aspects of this theory need to be explored.

Integrodifferential equations arise in many engineering and scientific disciplines, often as approximation to partial differential equations, which represent much of the continuum 
phenomena. Many forms of these equations are possible. Some of the applications are unsteady aerodynamics and aero elastic phenomena, visco elasticity, visco elastic panel in super sonic gas flow, fluid dynamics, electrodynamics of complex medium, many models of population growth, polymer rheology, neural network modeling, sandwich system identification, materials with fading memory, mathematical modeling of the diffusion of discrete particles in a turbulent fluid, heat conduction in materials with memory, theory of lossless transmission lines, theory of population dynamics, compartmental systems, nuclear reactors, and mathematical modeling of a hereditary phenomena. For details, see [23-29] and the references therein.

Integral boundary conditions have various applications in applied fields such as blood flow problems, chemical engineering, thermoelasticity, underground water flow, population dynamics, and so forth. For a detailed description of the integral boundary conditions, we refer the reader to a recent paper [30]. For more details of nonlocal and integral boundary conditions, see [31-37] and references therein.

In this paper, we consider the following boundary value problem for a nonlinear fractional integrodifferential equation with integral boundary conditions

$$
\begin{gathered}
{ }^{c} D^{q} x(t)=f(t, x(t),(x x)(t)), \quad 0<t<1,1<q \leq 2, \\
\alpha x(0)+\beta x^{\prime}(0)=\int_{0}^{1} q_{1}(x(s)) d s, \quad \alpha x(1)+\beta x^{\prime}(1)=\int_{0}^{1} q_{2}(x(s)) d s,
\end{gathered}
$$

where ${ }^{c} D$ is the Caputo fractional derivative, $f:[0,1] \times X \times X \rightarrow X$, for $\gamma:[0,1] \times[0,1] \rightarrow$ $[0, \infty)$,

$$
(x x)(t)=\int_{0}^{t} \gamma(t, s) x(s) d s
$$

$q_{1}, q_{2}: X \rightarrow X$ and $\alpha>0, \beta \geq 0$ are real numbers. Here, $(X,\|\cdot\|)$ is a Banach space and $C=$ $C([0,1], X)$ denotes the Banach space of all continuous functions from $[0,1] \rightarrow X$ endowed with a topology of uniform convergence with the norm denoted by $\|\cdot\|_{C}$.

\section{Preliminaries}

First of all, we recall some basic definitions $[15,18,20]$.

Definition 2.1. For a function $f:[0, \infty) \rightarrow \mathbb{R}$, the Caputo derivative of fractional order $q$ is defined as

$$
{ }^{c} D^{q} f(t)=\frac{1}{\Gamma(n-q)} \int_{0}^{t}(t-s)^{n-q-1} f^{(n)}(s) d s, \quad n-1<q<n, n=[q]+1,
$$

where $[q]$ denotes the integer part of the real number $q$. 
Definition 2.2. The Riemann-Liouville fractional integral of order $q$ is defined as

$$
I^{q} f(t)=\frac{1}{\Gamma(q)} \int_{0}^{t} \frac{f(s)}{(t-s)^{1-q}} d s, \quad q>0
$$

provided the integral exists.

Definition 2.3. The Riemann-Liouville fractional derivative of order $q$ for a function $f(t)$ is defined by

$$
D^{q} f(t)=\frac{1}{\Gamma(n-q)}\left(\frac{d}{d t}\right)^{n} \int_{0}^{t} \frac{f(s)}{(t-s)^{q-n+1}} d s, \quad n=[q]+1,
$$

provided the right hand side is pointwise defined on $(0, \infty)$.

In passing, we remark that the definition of Riemann-Liouville fractional derivative, which did certainly play an important role in the development of theory of fractional derivatives and integrals, could hardly produce the physical interpretation of the initial conditions required for the initial value problems involving fractional differential equations. The same applies to the boundary value problems of fractional differential equations. It was Caputo definition of fractional derivative which solved this problem. In fact, the Caputo derivative becomes the conventional $n$th derivative of the function $f(t)$ as $q \rightarrow n$ and the initial conditions for fractional differential equations retain the same form as that of ordinary differential equations with integer derivatives. Another difference is that the Caputo derivative for a constant is zero while the Riemann-Liouville fractional derivative of a constant is nonzero. For more details, see [20].

Lemma 2.4 (see [22]). For $q>0$, the general solution of the fractional differential equation ${ }^{c} D^{q} x(t)=0$ is given by

$$
x(t)=c_{0}+c_{1} t+c_{2} t^{2}+\cdots+c_{n-1} t^{n-1},
$$

where $c_{i} \in \mathbb{R}, i=0,1,2, \ldots, n-1(n=[q]+1)$.

In view of Lemma 2.4, it follows that

$$
I^{q c} D^{q} x(t)=x(t)+c_{0}+c_{1} t+c_{2} t^{2}+\cdots+c_{n-1} t^{n-1},
$$

for some $c_{i} \in \mathbb{R}, i=0,1,2, \ldots, n-1(n=[q]+1)$.

Now, we state a known result due to Krasnosel'skir [38] which is needed to prove the existence of at least one solution of (1.1).

Theorem 2.5. Let $M$ be a closed convex and nonempty subset of a Banach space X. Let $A, B$ be the operators such that (i) $A x+B y \in M$ whenever $x, y \in M$, (ii) $A$ is compact and continuous, (iii) $B$ is a contraction mapping. Then there exists $z \in M$ such that $z=A z+B z$. 
Lemma 2.6. For any $\zeta, \eta_{1}, \eta_{2} \in C[0,1]$, the unique solution of the boundary value problem

$$
\begin{gathered}
{ }^{c} D^{q} x(t)=\zeta(t), \quad 0<t<1,1<q \leq 2, \\
\alpha x(0)+\beta x^{\prime}(0)=\int_{0}^{1} \eta_{1}(s) d s, \quad \alpha x(1)+\beta x^{\prime}(1)=\int_{0}^{1} \eta_{2}(s) d s,
\end{gathered}
$$

is given by

$$
x(t)=\int_{0}^{1} G(t, s) \zeta(s) d s+\frac{1}{\alpha^{2}}\left[(\alpha(1-t)+\beta) \int_{0}^{1} \eta_{1}(s) d s+(\beta+\alpha t) \int_{0}^{1} \eta_{2}(s) d s\right],
$$

where $G(t, s)$ is the Green's function given by

$$
G(t, s)= \begin{cases}\frac{\alpha(t-s)^{q-1}+(\beta-\alpha t)(1-s)^{q-1}}{\alpha \Gamma(q)}+\frac{\beta(\beta-\alpha t)(1-s)^{q-2}}{\alpha^{2} \Gamma(q-1)}, & s \leq t, \\ \frac{(\beta-\alpha t)(1-s)^{q-1}}{\alpha \Gamma(q)}+\frac{\beta(\beta-\alpha t)(1-s)^{q-2}}{\alpha^{2} \Gamma(q-1)}, & t \leq s .\end{cases}
$$

Proof. Using (2.5), for some constants $b_{1}, b_{2} \in \mathbb{R}$, we have

$$
x(t)=I^{q} \zeta(t)-b_{1}-b_{2} t=\int_{0}^{t} \frac{(t-s)^{q-1}}{\Gamma(q)} \zeta(s) d s-b_{1}-b_{2} t
$$

In view of the relations ${ }^{c} D^{q} I^{q} x(t)=x(t)$ and $I^{q} I^{p} x(t)=I^{q+p} x(t)$ for $q, p>0, x \in L(0,1)$, we obtain

$$
x^{\prime}(t)=\int_{0}^{t} \frac{(t-s)^{q-2}}{\Gamma(q-1)} \zeta(s) d s-b_{2}
$$

Applying the boundary conditions for (2.6), we find that

$$
\begin{aligned}
b_{1}= & \frac{1}{\alpha^{2}}\left[\beta \int_{0}^{1} \eta_{2}(s) d s-(\beta+\alpha) \int_{0}^{1} \eta_{1}(s) d s\right]-\frac{\beta}{\alpha \Gamma(q)} \int_{0}^{1}(1-s)^{q-1} \zeta(s) d s \\
& -\frac{\beta^{2}}{\alpha^{2} \Gamma(q-1)} \int_{0}^{1}(1-s)^{q-2} \zeta(s) d s, \\
b_{2}= & \frac{1}{\alpha}\left[\int_{0}^{1} \eta_{1}(s) d s-\int_{0}^{1} \eta_{2}(s) d s\right]+\frac{1}{\Gamma(q)} \int_{0}^{1}(1-s)^{q-1} \zeta(s) d s \\
& +\frac{\beta}{\alpha \Gamma(q-1)} \int_{0}^{1}(1-s)^{q-2} \zeta(s) d s .
\end{aligned}
$$


Thus, the unique solution of (2.6) is

$$
\begin{aligned}
x(t)= & \int_{0}^{t}\left[\frac{\alpha(t-s)^{q-1}+(\beta-\alpha t)(1-s)^{q-1}}{\alpha \Gamma(q)}+\frac{\beta(\beta-\alpha t)(1-s)^{q-2}}{\alpha^{2} \Gamma(q-1)}\right] \zeta(s) d s \\
& +\int_{t}^{1}\left[\frac{(\beta-\alpha t)(1-s)^{q-1}}{\alpha \Gamma(q)}+\frac{\beta(\beta-\alpha t)(1-s)^{q-2}}{\alpha^{2} \Gamma(q-1)}\right] \zeta(s) d s \\
& +\frac{1}{\alpha^{2}}\left[(\alpha(1-t)+\beta) \int_{0}^{1} \eta_{1}(s) d s+(\beta+\alpha t) \int_{0}^{1} \eta_{2}(s) d s\right] \\
= & \int_{0}^{1} G(t, s) \zeta(s) d s+\frac{1}{\alpha^{2}}\left[(\alpha(1-t)+\beta) \int_{0}^{1} \eta_{1}(s) d s+(\beta+\alpha t) \int_{0}^{1} \eta_{2}(s) d s\right],
\end{aligned}
$$

where $G(t, s)$ is given by $(2.8)$. This completes the proof.

\section{Main Results}

Theorem 3.1. Assume that $f:[0,1] \times X \times X \rightarrow X$ is jointly continuous and maps bounded subsets of $[0,1] \times X \times X$ into relatively compact subsets of $X, \gamma:[0,1] \times[0,1] \rightarrow[0, \infty)$ is continuous with $\gamma_{0}=\max \{\gamma(t, s):(t, s) \in[0,1] \times[0,1]\}$, and $q_{1}, q_{2}: X \rightarrow X$ are continuous functions. Further, there exist positive constants $L_{1}, \bar{L}_{1}, L_{2}, L_{3}, M_{2}, M_{3}$ such that

$\left(\mathrm{A}_{1}\right)\|f(t, x(t),(\chi x)(t))-f(t, y(t),(x y)(t))\| \leq L_{1}\|x-y\|+\bar{L}_{1}\|x x-x y\|$, for all $t \in[0,1]$, $x, y \in X$,

$\left(\mathrm{A}_{2}\right)\left\|q_{1}(x)-q_{1}(y)\right\| \leq L_{2}\|x-y\|,\left\|q_{2}(x)-q_{2}(y)\right\| \leq L_{3}\|x-y\|$ with $\left\|q_{1}(x)\right\| \leq M_{2},\left\|q_{2}(x)\right\| \leq$ $M_{3}$, for all $x, y \in X$.

Then the boundary value problem (1.1) has a unique solution provided

$$
\left(L_{1}+\gamma_{0} \bar{L}_{1}\right)\left[\frac{\beta+2 \alpha}{\alpha \Gamma(q+1)}+\frac{\beta^{2}+\alpha \beta}{\alpha^{2} \Gamma(q)}\right]+\frac{\beta+\alpha}{\alpha^{2}}\left(L_{2}+L_{3}\right)<1
$$

with

$$
L_{1}+\gamma_{0} \bar{L}_{1} \leq \frac{1}{2}\left[\frac{\beta+2 \alpha}{\alpha \Gamma(q+1)}+\frac{\beta^{2}+\alpha \beta}{\alpha^{2} \Gamma(q)}\right]^{-1}
$$

Proof. Define $\digamma: C \rightarrow C$ by

$$
\begin{aligned}
(\digamma x)(t)= & \frac{1}{\Gamma(q)} \int_{0}^{t}(t-s)^{q-1} f(s, x(s),(\chi x)(s)) d s \\
& +\int_{0}^{1}\left[\frac{(\beta-\alpha t)(1-s)^{q-1}}{\alpha \Gamma(q)}+\frac{\beta(\beta-\alpha t)(1-s)^{q-2}}{\alpha^{2} \Gamma(q-1)}\right] f(s, x(s),(\chi x)(s)) d s \\
& +\frac{1}{\alpha^{2}}\left[(\alpha(1-t)+\beta) \int_{0}^{1} q_{1}(x(s)) d s+(\beta+\alpha t) \int_{0}^{1} q_{2}(x(s)) d s\right], \quad t \in[0,1] .
\end{aligned}
$$


Setting $\sup _{t \in[0,1]}\|f(t, 0,0)\|=M_{1}$ (by the assumption on $f$ ) and Choosing

$$
r \geq 2\left[M_{1}\left(\frac{\beta+2 \alpha}{\alpha \Gamma(q+1)}+\frac{\beta^{2}+\alpha \beta}{\alpha^{2} \Gamma(q)}\right)+\frac{\alpha+\beta}{\alpha^{2}}\left(M_{2}+M_{3}\right)\right]
$$

we show that $\digamma B_{r} \subset B_{r}$, where $B_{r}=\{x \in C:\|x\| \leq r\}$. For $x \in B_{r}$, we have

$$
\begin{aligned}
& \|(\digamma x)(t)\| \leq \frac{1}{\Gamma(q)} \int_{0}^{t}(t-s)^{q-1}\|f(s, x(s),(\chi x)(s))\| d s \\
& +\int_{0}^{1}|\beta-\alpha t|\left[\frac{(1-s)^{q-1}}{\alpha \Gamma(q)}+\frac{\beta(1-s)^{q-2}}{\alpha^{2} \Gamma(q-1)}\right]\|f(s, x(s),(\chi x)(s))\| d s \\
& +\frac{\alpha+\beta}{\alpha^{2}}\left(M_{2}+M_{3}\right) \\
& \leq \frac{1}{\Gamma(q)} \int_{0}^{t}(t-s)^{q-1}[\|f(s, x(s),(\chi x)(s))-f(s, 0,0)\|+\|f(s, 0,0)\|] d s \\
& +\int_{0}^{1}|\beta-\alpha t|\left[\frac{(1-s)^{q-1}}{\alpha \Gamma(q)}+\frac{\beta(1-s)^{q-2}}{\alpha^{2} \Gamma(q-1)}\right] \\
& \times[\|f(s, x(s),(\chi x)(s))-f(s, 0,0)\|+\|f(s, 0,0)\|] d s+\frac{\alpha+\beta}{\alpha^{2}}\left(M_{2}+M_{3}\right) \\
& \leq\left(\left(L_{1}+\gamma_{0} \bar{L}_{1}\right) r+M_{1}\right)\left[\frac{1}{\Gamma(q)} \int_{0}^{t}(t-s)^{q-1} d s\right. \\
& \left.+\int_{0}^{1}|\beta-\alpha t|\left(\frac{(1-s)^{q-1}}{\alpha \Gamma(q)}+\frac{\beta(1-s)^{q-2}}{\alpha^{2} \Gamma(q-1)}\right) d s\right] \\
& +\frac{\alpha+\beta}{\alpha^{2}}\left(M_{2}+M_{3}\right) \\
& =\left(\left(L_{1}+\gamma_{0} \bar{L}_{1}\right) r+M_{1}\right)\left[\frac{t^{q}}{\Gamma(q+1)}+|\beta-\alpha t|\left(\frac{1}{\alpha \Gamma(q+1)}+\frac{\beta}{\alpha^{2} \Gamma(q)}\right)\right] \\
& +\frac{\alpha+\beta}{\alpha^{2}}\left(M_{2}+M_{3}\right) \\
& \leq\left(L_{1}+\gamma_{0} \bar{L}_{1}\right)\left[\frac{2 \alpha+\beta}{\alpha \Gamma(q+1)}+\frac{\beta^{2}+\alpha \beta}{\alpha^{2} \Gamma(q)}\right] r \\
& +M_{1}\left[\frac{2 \alpha+\beta}{\alpha \Gamma(q+1)}+\frac{\beta^{2}+\alpha \beta}{\alpha^{2} \Gamma(q)}\right]+\frac{\alpha+\beta}{\alpha^{2}}\left(M_{2}+M_{3}\right) \leq r .
\end{aligned}
$$


Now, for $x, y \in C$ and for each $t \in[0,1]$, we obtain

$$
\begin{aligned}
\|(\digamma x)(t) & -(\digamma y)(t) \| \\
\leq & \frac{1}{\Gamma(q)} \int_{0}^{t}(t-s)^{q-1}\|f(s, x(s),(x x)(s))-f(s, y(s),(x y)(s))\| d s \\
& +\int_{0}^{1}|\beta-\alpha t|\left[\frac{(1-s)^{q-1}}{\alpha \Gamma(q)}+\frac{\beta(1-s)^{q-2}}{\alpha^{2} \Gamma(q-1)}\right]\|f(s, x(s),(x x)(s))-f(s, y(s),(x y)(s))\| d s \\
& +\frac{\alpha+\beta}{\alpha^{2}}\left[\int_{0}^{1}\left\|q_{1}(x(s))-q_{1}(y(s))\right\| d s+\int_{0}^{1}\left\|q_{2}(x(s))-q_{2}(y(s))\right\| d s\right] \\
\leq & \left(L_{1}+\gamma_{0} \bar{L}_{1}\right)\|x-y\|_{C}\left[\frac{t^{q}}{\Gamma(q+1)}+|\beta-\alpha t|\left(\frac{1}{\alpha \Gamma(q+1)}+\frac{\beta}{\alpha^{2} \Gamma(q)}\right)\right] \\
& +\frac{\alpha+\beta}{\alpha^{2}}\left(L_{2}+L_{3}\right)\|x-y\|_{C} \\
\leq & \left\{\left(L_{1}+\gamma_{0} \bar{L}_{1}\right)\left[\frac{2 \alpha+\beta}{\alpha \Gamma(q+1)}+\frac{\left.\beta^{2}+\alpha \beta\right)}{\alpha^{2} \Gamma(q)}\right]+\frac{\alpha+\beta}{\alpha^{2}}\left(L_{2}+L_{3}\right)\right\}\|x-y\|_{C} \\
\leq & \Lambda_{\alpha, \beta, q, \gamma_{0}, L_{1}, \bar{L}_{1}, L_{2}, L_{3}}\|x-y\|_{C},
\end{aligned}
$$

where

$$
\Lambda_{\alpha, \beta, q, \gamma_{0}, L_{1}, \bar{L}_{1}, L_{2}, L_{3}}=\left(L_{1}+\gamma_{0} \bar{L}_{1}\right)\left[\frac{2 \alpha+\beta}{\alpha \Gamma(q+1)}+\frac{\beta^{2}+\alpha \beta}{\alpha^{2} \Gamma(q)}\right]+\frac{\alpha+\beta}{\alpha^{2}}\left(L_{2}+L_{3}\right)
$$

which depends only on the parameters involved in the problem. As $\Lambda_{\alpha, \beta, q, L_{1}, \bar{L}_{1}, L_{2}, L_{3}}<1$, therefore $\digamma$ is a contraction. Thus, the conclusion of the theorem follows by the contraction mapping principle.

Theorem 3.2. Assume that $\left(A_{1}\right)-\left(A_{2}\right)$ hold with $\|f(t, x(t),(\chi x)(t))\| \leq \mu(t)$, for all $\left(t, x, \chi^{x}\right) \in$ $[0,1] \times X \times X$, where $\mu \in L^{1}\left([0,1], R^{+}\right)$and

$$
\left(L_{1}+\gamma_{0} \bar{L}_{1}\right)\left(\frac{\alpha+\beta}{\alpha \Gamma(q+1)}+\frac{\beta^{2}+\alpha \beta}{\alpha^{2} \Gamma(q)}\right)+\frac{\alpha+\beta}{\alpha^{2}}\left(L_{2}+L_{3}\right)<1
$$

Then the boundary value problem (1.1) has at least one solution on $[0,1]$.

Proof. Let us fix

$$
r \geq\|\mu\|_{L^{1}}\left[\frac{2 \alpha+\beta}{\alpha \Gamma(q+1)}+\frac{\beta^{2}+\alpha \beta}{\alpha^{2} \Gamma(q)}\right]+\frac{\alpha+\beta}{\alpha^{2}}\left(M_{2}+M_{3}\right),
$$


and consider $B_{r}=\{x \in C:\|x\| \leq r\}$. We define the operators $\Phi$ and $\Psi$ on $B_{r}$ as

$$
\begin{aligned}
(\Phi x)(t)= & \frac{1}{\Gamma(q)} \int_{0}^{t}(t-s)^{q-1} f(s, x(s),(\chi x)(s)) d s \\
(\Psi x)(t)= & \int_{0}^{1}\left[\frac{(\beta-\alpha t)(1-s)^{q-1}}{\alpha \Gamma(q)}+\frac{\beta(\beta-\alpha t)(1-s)^{q-2}}{\alpha^{2} \Gamma(q-1)}\right] f(s, x(s),(\chi x)(s)) d s \\
& +\frac{1}{\alpha^{2}}\left[(\alpha(1-t)+\beta) \int_{0}^{1} q_{1}(x(s)) d s+(\beta+\alpha t) \int_{0}^{1} q_{2}(x(s)) d s\right] .
\end{aligned}
$$

For $x, y \in B_{r}$, we find that

$$
\|\Phi x+\Psi y\| \leq\|\mu\|_{L^{1}}\left[\frac{2 \alpha+\beta}{\alpha \Gamma(q+1)}+\frac{\beta^{2}+\alpha \beta}{\alpha^{2} \Gamma(q)}\right]+\frac{\alpha+\beta}{\alpha^{2}}\left(M_{2}+M_{3}\right) \leq r .
$$

Thus, $\Phi x+\Psi y \in B_{r}$. It follows from the assumption $\left(\mathrm{A}_{1}\right),\left(\mathrm{A}_{2}\right)$ that $\Psi$ is a contraction mapping for

$$
\left(L_{1}+\gamma_{0} \bar{L}_{1}\right)\left(\frac{\alpha+\beta}{\alpha \Gamma(q+1)}+\frac{\beta^{2}+\alpha \beta}{\alpha^{2} \Gamma(q)}\right)+\frac{\alpha+\beta}{\alpha^{2}}\left(L_{2}+L_{3}\right)<1 .
$$

Continuity of $f$ implies that the operator $\Phi$ is continuous. Also, $\Phi$ is uniformly bounded on $B_{r}$ as

$$
\|\Phi x\| \leq \frac{\|\mu\|_{L^{1}}}{\Gamma(q+1)}
$$

Now we prove the compactness of the operator $\Phi$. In view of $\left(A_{1}\right)$, we define $\sup _{(t, x, x x) \in \Omega}\|f(s, x(s),(\chi x)(s))\|=f_{\max }, \Omega=[0,1] \times B_{r} \times B_{r}$, and consequently we have

$$
\begin{aligned}
& \left\|(\Phi x)\left(t_{1}\right)-(\Phi x)\left(t_{2}\right)\right\| \\
& =\| \frac{1}{\Gamma(q)} \int_{0}^{t_{1}}\left[\left(t_{2}-s\right)^{q-1}-\left(t_{1}-s\right)^{q-1}\right] f(s, x(s),(\chi x)(s)) d s \\
& \quad+\int_{t_{1}}^{t_{2}}\left(t_{2}-s\right)^{q-1} f(s, x(s)) d s \| \\
& \leq \frac{f_{\max }}{\Gamma(q+1)}\left|2\left(t_{2}-t_{1}\right)^{q}+t_{1}^{q}-t_{2}^{q}\right|,
\end{aligned}
$$

which is independent of $x$. So $\Phi$ is relatively compact on $B_{r}$. Hence, By Arzela Ascoli Theorem, $\Phi$ is compact on $B_{r}$. Thus all the assumptions of Theorem 2.5 are satisfied and 
the conclusion of Theorem 2.5 implies that the boundary value problem (1.1) has at least one solution on $[0,1]$.

Example 3.3. Consider the following boundary value problem:

$$
\begin{gathered}
{ }^{c} D^{q} x(t)=\frac{1}{(t+7)^{2}} \frac{|x|}{1+|x|}+\int_{0}^{t} \frac{e^{-(s-t)}}{49} x(s) d s, \quad t \in[0,1], 1<q \leq 2, \\
x(0)+x^{\prime}(0)=\int_{0}^{1} \frac{|x(s)|}{5+|x(s)|} d s, \quad x(1)+x^{\prime}(1)=\int_{0}^{1} \frac{|x(s)|}{7+|x(s)|} d s .
\end{gathered}
$$

Here, $f(t, x)=\left(1 /(t+7)^{2}\right)(|x| /(1+|x|)), \gamma(t, s)=e^{-(s-t)} / 49, q_{1}(x)=|x| /(5+|x|), q_{2}(x)=$ $|x| /(7+|x|), \alpha=1, \beta=1$. As $\|f(t, x, x x)-f(t, y, x y)\| \leq(1 / 49)\|x-y\|+\|x x-x y\|, \| q_{1}(x)-$ $q_{1}(y)\|\leq(1 / 5)\| x-y\|,\| q_{2}(x)-q_{2}(y)\|\leq(1 / 7)\| x-y \|$, therefore, $\left(\mathrm{A}_{1}\right)$ and $\left(\mathrm{A}_{2}\right)$ are satisfied with $L_{1}=1 / 49, \bar{L}_{1}=1, \gamma_{0}=((e-1) / 49) L_{2}=1 / 5, L_{3}=1 / 7$. Further,

$$
\left(L_{1}+\gamma_{0} \bar{L}_{1}\right)\left[\frac{\beta+2 \alpha}{\alpha \Gamma(q+1)}+\frac{\beta^{2}+\alpha \beta}{\alpha^{2} \Gamma(q)}\right]+\frac{\beta+\alpha}{\alpha^{2}}\left(L_{2}+L_{3}\right)<1 \Longleftrightarrow \frac{e}{49}\left(\frac{3}{\Gamma(q+1)}+\frac{2}{\Gamma(q)}\right)<\frac{11}{35} .
$$

Thus, by Theorem 3.1, the boundary value problem (3.15) has a unique solution on $[0,1]$.

\section{Acknowledgments}

The authors are grateful to the anonymous referee for his/her valuable suggestions that led to the improvement of the original manuscript. The research of J. J. Nieto has been partially supported by Ministerio de Educacion y Ciencia and FEDER, Project MTM2007-61724, and by Xunta de Galicia and FEDER, project PGIDIT05PXIC20702PN.

\section{References}

[1] B. Ahmad and J. J. Nieto, "Existence results for a coupled system of nonlinear fractional differential equations with three-point boundary conditions," preprint.

[2] B. Ahmad and S. Sivasundaram, "Existence and uniqueness results for nonlinear boundary value problems of fractional differential equations with separated boundary conditions," to appear in Dynamic Systems and Applications.

[3] D. Araya and C. Lizama, "Almost automorphic mild solutions to fractional differential equations," Nonlinear Analysis: Theory, Methods \& Applications, vol. 69, no. 11, pp. 3692-3705, 2008.

[4] Z. Bai and H. Lü, "Positive solutions for boundary value problem of nonlinear fractional differential equation," Journal of Mathematical Analysis and Applications, vol. 311, no. 2, pp. 495-505, 2005.

[5] M. Belmekki, J. J. Nieto, and R. Rodríguez-López, "Existence of periodic solution for a nonlinear fractional differential equation," preprint.

[6] M. Benchohra, S. Hamani, J. J. Nieto, and B. A. Slimani, "Existence results for differential inclusions with fractional order and impulses," preprint.

[7] B. Bonilla, M. Rivero, L. Rodríguez-Germá, and J. J. Trujillo, "Fractional differential equations as alternative models to nonlinear differential equations," Applied Mathematics and Computation, vol. 187, no. 1, pp. 79-88, 2007.

[8] Y.-K. Chang and J. J. Nieto, "Some new existence results for fractional differential inclusions with boundary conditions," Mathematical and Computer Modelling, vol. 49, no. 3-4, pp. 605-609, 2009. 
[9] V. Gafiychuk, B. Datsko, and V. Meleshko, "Mathematical modeling of time fractional reactiondiffusion systems," Journal of Computational and Applied Mathematics, vol. 220, no. 1-2, pp. 215-225, 2008.

[10] V. Daftardar-Gejji, "Positive solutions of a system of non-autonomous fractional differential equations," Journal of Mathematical Analysis and Applications, vol. 302, no. 1, pp. 56-64, 2005.

[11] V. Daftardar-Gejji and S. Bhalekar, "Boundary value problems for multi-term fractional differential equations," Journal of Mathematical Analysis and Applications, vol. 345, no. 2, pp. 754-765, 2008.

[12] M. El-Shahed, "Positive solutions for boundary value problem of nonlinear fractional differential equation," Abstract and Applied Analysis, vol. 2007, Article ID 10368, 8 pages, 2007.

[13] R. W. Ibrahim and M. Darus, "Subordination and superordination for univalent solutions for fractional differential equations," Journal of Mathematical Analysis and Applications, vol. 345, no. 2, pp. 871-879, 2008.

[14] H. Jafari and S. Seifi, "Homotopy analysis method for solving linear and nonlinear fractional diffusion-wave equation," Communications in Nonlinear Science and Numerical Simulation, vol. 14, no. 5, pp. 2006-2012, 2009.

[15] A. A. Kilbas, H. M. Srivastava, and J. J. Trujillo, Theory and Applications of Fractional Differential Equations, vol. 204 of North-Holland Mathematics Studies, Elsevier Science B.V., Amsterdam, The Netherlands, 2006.

[16] S. Ladaci, J. J. Loiseau, and A. Charef, "Fractional order adaptive high-gain controllers for a class of linear systems," Communications in Nonlinear Science and Numerical Simulation, vol. 13, no. 4, pp. 707-714, 2008.

[17] M. P. Lazarević, "Finite time stability analysis of $\mathrm{PD}^{\alpha}$ fractional control of robotic time-delay systems," Mechanics Research Communications, vol. 33, no. 2, pp. 269-279, 2006.

[18] I. Podlubny, Fractional Differential Equations, vol. 198 of Mathematics in Science and Engineering, Academic Press, San Diego, Calif, USA, 1999.

[19] S. Z. Rida, H. M. El-Sherbiny, and A. A. M. Arafa, "On the solution of the fractional nonlinear Schrödinger equation," Physics Letters A, vol. 372, no. 5, pp. 553-558, 2008.

[20] S. G. Samko, A. A. Kilbas, and O. I. Marichev, Fractional Integrals and Derivatives: Theory and Applications, Gordon and Breach Science, Yverdon, Switzerland, 1993.

[21] V. Varlamov, "Differential and integral relations involving fractional derivatives of Airy functions and applications," Journal of Mathematical Analysis and Applications, vol. 348, no. 1, pp. 101-115, 2008.

[22] S. Zhang, "Positive solutions for boundary-value problems of nonlinear fractional differential equations," Electronic Journal of Differential Equations, vol. 2006, no. 36, pp. 1-12, 2006.

[23] B. Ahmad and S. Sivasundaram, "Some existence results for fractional integrodifferential equations with nonlinear conditions," Communications in Applied Analysis, vol. 12, pp. 107-112, 2008.

[24] B. Ahmad and B. S. Alghamdi, "Approximation of solutions of the nonlinear Duffing equation involving both integral and non-integral forcing terms with separated boundary conditions," Computer Physics Communications, vol. 179, no. 6, pp. 409-416, 2008.

[25] B. Ahmad, "On the existence of $T$-periodic solutions for Duffing type integro-differential equations with $p$-Laplacian," Lobachevskii Journal of Mathematics, vol. 29, no. 1, pp. 1-4, 2008.

[26] Y. K. Chang and J. J. Nieto, “Existence of solutions for impulsive neutral integrodifferential inclusions with nonlocal initial conditions via fractional operators," to appear in Numerical Functional Analysis and Optimization.

[27] Z. Luo and J. J. Nieto, "New results for the periodic boundary value problem for impulsive integrodifferential equations," Nonlinear Analysis: Theory, Methods $\mathcal{E}$ Applications, vol. 70, no. 6, pp. 2248-2260, 2009.

[28] S. Mesloub, “On a mixed nonlinear one point boundary value problem for an integrodifferential equation," Boundary Value Problems, vol. 2008, Article ID 814947, 8 pages, 2008.

[29] J. J. Nieto and R. Rodríguez-López, "New comparison results for impulsive integro-differential equations and applications," Journal of Mathematical Analysis and Applications, vol. 328, no. 2, pp. 1343 1368, 2007.

[30] B. Ahmad, A. Alsaedi, and B. S. Alghamdi, "Analytic approximation of solutions of the forced Duffing equation with integral boundary conditions," Nonlinear Analysis: Real World Applications, vol. 9, no. 4, pp. 1727-1740, 2008.

[31] B. Ahmad and A. Alsaedi, "Existence of approximate solutions of the forced Duffing equation with discontinuous type integral boundary conditions," Nonlinear Analysis: Real World Applications, vol. 10, no. 1 , pp. 358-367, 2009. 
[32] M. Benchohra, S. Hamani, and J. J. Nieto, "The method of upper and lower solutions for second order differential inclusions with integral boundary conditions," Rocky Mountain Journal of Mathematics. In press.

[33] A. Boucherif, "Second-order boundary value problems with integral boundary conditions," Nonlinear Analysis: Theory, Methods \& Applications, vol. 70, no. 1, pp. 364-371, 2009.

[34] Y.-K. Chang, J. J. Nieto, and W.-S. Li, “On impulsive hyperbolic differential inclusions with nonlocal initial conditions," Journal of Optimization Theory and Applications, vol. 140, no. 3, pp. 431-442, 2009.

[35] Y. K. Chang, J. J. Nieto, and W. S. Li, "Controllability of semilinear differential systems with nonlocal initial conditions in Banach spaces," to appear in Journal of Optimization Theory and Applications.

[36] M. Feng, B. Du, and W. Ge, "Impulsive boundary value problems with integral boundary conditions and one-dimensional $p$-Laplacian," Nonlinear Analysis: Theory, Methods \& Applications. In press.

[37] Z. Yang, "Existence of nontrivial solutions for a nonlinear Sturm-Liouville problem with integral boundary conditions," Nonlinear Analysis: Theory, Methods \& Applications, vol. 68, no. 1, pp. 216-225, 2008.

[38] M. A. Krasnosel'skiľ, "Two remarks on the method of successive approximations," Uspekhi Matematicheskikh Nauk, vol. 10, no. 1(63), pp. 123-127, 1955. 